\title{
EXPERT CONSENSUS DOCUMENT \\ Defining the major health modifiers causing atrial fibrillation: a roadmap to underpin personalized prevention and treatment
}

Larissa Fabritz ${ }^{1,2 *}$, Eduard Guasch ${ }^{3,4 *}$, Charalambos Antoniades ${ }^{5}$, Isabel Bardinet ${ }^{6}$, Gerlinde Benninger ${ }^{7}$, Tim R. Betts ${ }^{5}$, Eva Brand ${ }^{2}$, Günter Breithardt', ${ }^{2,}$, Gabriela Bucklar-Suchankova ${ }^{8}$, A. John Camm ${ }^{9}, 10$, David Cartlidge ${ }^{11}$, Barbara Casadei ${ }^{5}$, Winnie W. L. Chua ${ }^{1}$, Harry J. G. M. Crijns ${ }^{12,13}$, Jon Deeks' ${ }^{1}$, Stéphane Hatem ${ }^{14}$, Françoise Hidden-Lucet ${ }^{14}$, Stefan Kääb ${ }^{15,16}$, Nikos Maniadakis ${ }^{17}$, Stephan Martin ${ }^{6}$, Lluis Mont ${ }^{3,4}$, Holger Reinecke ${ }^{2}$, Moritz F. Sinner ${ }^{15}$, Ulrich Schotten ${ }^{12,13}$, Taunton Southwood ${ }^{1}$, Monika Stoll'12,13, Panos Vardas ${ }^{18}$, Reza Wakili'15,16, Andy West ${ }^{11}$, André Ziegler ${ }^{8}$ and Paulus Kirchhof ${ }^{1,2,7,19,20}$

Abstract | Despite remarkable advances in antiarrhythmic drugs, ablation procedures, and stroke-prevention strategies, atrial fibrillation (AF) remains an important cause of death and disability in middle-aged and elderly individuals. Unstructured management of patients with AF sharply contrasts with our detailed, although incomplete, knowledge of the mechanisms that cause AF and its complications. Altered calcium homeostasis, atrial fibrosis and ageing, ion-channel dysfunction, autonomic imbalance, fat-cell infiltration, and oxidative stress, in addition to a susceptible genetic background, contribute to the promotion, maintenance, and progression of AF. However, clinical management of patients with $\mathrm{AF}$ is currently guided by stroke risk parameters, AF pattern, and symptoms. In response to this apparent disconnect between the known pathophysiology of AF and clinical management, we propose a roadmap to develop a set of clinical markers that reflect the major causes of AF in patients. Thereby, the insights into the mechanisms causing AF will be transformed into a format that can underpin future personalized strategies to prevent and treat $\mathrm{AF}$, ultimately informing better patient care.

'Institute of Cardiovascular Sciences, University of Birmingham, Edgbaston, Birmingham B15 2TT, UK. ${ }^{2}$ Department of Cardiovascular Medicine, Division of Rhythmology, University Hospital Münster, Schlossplatz 2,

48149 Munich, Germany.

${ }^{*}$ These authors are joint first authors.

Correspondence to L.F.

l.fabritz@bham.ac.uk

doi:10.1038/nrcardio2015.194

Published online 24 Dec 2015
Despite the important advances that have enabled better stroke prevention in atrial fibrillation (AF) and more effective maintenance of sinus rhythm over the past decades, a large unmet need to improve the prevention and treatment of AF remains. Mortality for AF remains at $3.5 \%$ per year, and death is often experienced as sudden death or as a result of heart failure ${ }^{1,2}$. Each year, approximately $20 \%$ of patients with AF need to be hospitalized ${ }^{3,4}$, and stroke occurs in $1.5 \%$ of patients with AF who are receiving anticoagulant drugs $^{5}$. Furthermore, more than half of the patients with $\mathrm{AF}$ are symptomatic despite adequate anticoagulation and rate control ${ }^{4,6}$. In view of the projected increase in the incidence and prevalence of $\mathrm{AF}^{7-9}$, as well as the substantial burden of death and disability that is still associated with this condition ${ }^{10}$, the status quo is unacceptable.

Current management of patients with AF comprises treatment of the accompanying cardiovascular conditions, oral anticoagulation, rate control — with medications that slow atrioventricular nodal recovery or, rarely, with atrioventricular nodal ablation - and rhythm-control therapy with antiarrhythmic drugs, electrical cardioversion, catheter ablation or, at times, AF surgery ${ }^{11,12}$. Unfortunately, most of these current approaches are disconnected from our understanding of the major mechanisms that cause $\mathrm{AF}^{1,13,14}$ (BOX 1). AF is a heterogeneous condition with multiple aetiologies 


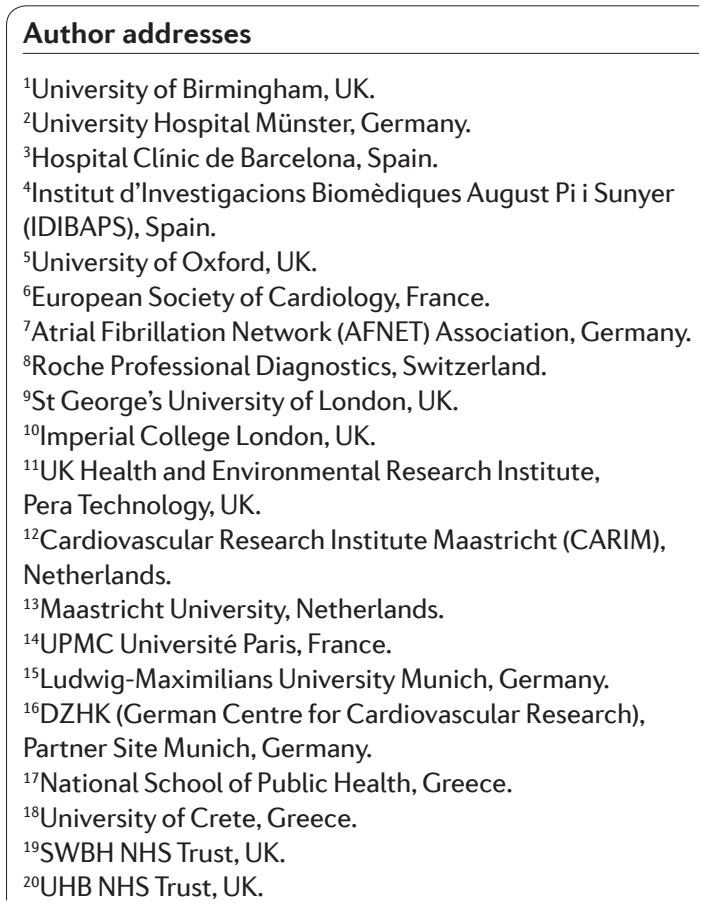

and mechanisms, and which presents with a variety of symptoms and evolution patterns ${ }^{2,15-23}$. However, none of the current management decisions considers the variable pathophysiology of AF, thereby ignoring the opportunity for tailored, personalized approaches that target the major drivers for $\mathrm{AF}^{24}$.

At present, anticoagulation is recommended on the basis of clinical risk scores for stroke, irrespective of the type or cause of AF. Rate-control therapy is titrated on the basis of resting heart rate and symptoms, without individualized discrimination between patients. Only symptoms and, to some degree, the temporal pattern of AF, comorbidities, and life expectancy, are considered when making the decision about rhythm-control ther$\mathrm{apy}^{25}$. Even the 'simple' distinction between paroxysmal and chronic AF is often incorrect, and correlates poorly with the real AF burden ${ }^{26,27}$.

The indiscriminate use of upstream AF therapies has led to some disappointment. Experimental

\footnotetext{
Box 1 | Disconnect between known mechanism of AF and management

At present, a disconnect exists between the known mechanisms of atrial fibrillation (AF), and the current management of AF, which is based on a useful, but weak, estimation scheme of the risk of stroke and on AF duration and symptoms (see the figure).

\begin{tabular}{|l|}
\hline Known pathophysiological \\
mechanisms \\
- Atrial fibrosis \\
- Abnormal $\mathrm{Ca}^{2+}$ homeostasis \\
- Ion-channel dysfunction \\
(genetic or acquired) \\
- Autonomic dysfunction \\
- Increased oxidative stress \\
- microRNA-mediated dysregulation \\
- Paracrine fat-cell activity \\
\hline
\end{tabular}

$\left\{\begin{array}{l}\text { Current management strategies } \\ \text { Therapy of concomitant conditions } \\ \text { in all patients } \\ \text { Anticoagulation stratified by } \\ \text { stroke risk factors } \\ \text { Rate control in all patients, } \\ \text { titrated to rate } \\ \text { Rhythm control in symptomatic } \\ \text { patients, partially based on AF duration } \\ \text { or AF pattern }\end{array}\right.$
}

and observational clinical data supported reninangiotensin-aldosterone blockers in AF prevention scheme ${ }^{28,29}$, but subsequent large randomized trials did not demonstrate a clinical benefit in patients without an established indication for these substances ${ }^{30,31}$. Drugs with anti-inflammatory and antioxidative properties that were tested for the prevention of AF in several settings showed mixed outcomes ${ }^{23,32-35}$. Finding markers for the major disease mechanisms - or 'health modifiers' - causing AF in a given patient would enable some of these treatment modalities to be used successfully. Furthermore, if information on the individual drivers for AF were available, personalized, preventive, and therapeutic strategies against this major threat to healthy ageing could be developed ${ }^{36}$.

In this Consensus Statement, we critically assess how the current knowledge on the mechanisms of AF is identified in daily clinical practice, and the extent to which it is translated into therapeutic improvements. To bridge the current gap between basic knowledge and clinical management, we propose a series of steps to build a clinically useful, mechanistic classification of $\mathrm{AF}$, and describe the starting point for developing a set of clinical markers for the major health modifiers causing $\mathrm{AF}$.

\section{Guidance through AF characterization}

In recognition of the current shortfalls in AF management, several research groups and consensus panels have proposed methods and approaches for improved characterization of the causes of AF in patients. Some groups have recommended the use of cardiac MRIdetected left atrial delayed gadolinium enhancement ${ }^{37-39}$ for the identification of patients in whom AF is likely to recur after catheter ablation ${ }^{38,40,41}$, although an external validation of the feasibility of this technique is needed ${ }^{42}$. The CHARGE AF consortium identified a set of clinical parameters and biomarkers that could be used to predict AF incidence during follow-up, but did not provide information on the most appropriate prevention or therapy approach ${ }^{43}$. Similarly, the CHADS, score predicts new-onset AF incidence ${ }^{44}$, but does not add information on the causes or the mechanisms involved. Delegates at the $4^{\text {th }}$ AFNET/EHRA consensus conference proposed a classification of AF that reflected the different disease mechanisms. The proposed AF types were: monogenic $\mathrm{AF}$, focally induced $\mathrm{AF}$, postoperative $\mathrm{AF}$, valvular $\mathrm{AF}, \mathrm{AF}$ in the elderly, polygenic $\mathrm{AF}$, and unclassified $\mathrm{AF}^{45,46}$. Unfortunately, however, prospective validation of such a classification is currently lacking, resulting in many patients being classified as 'other.' Moreover, many patients, including those with unclassified AF, will show overlapping mechanisms of AF according to this expert consensus.

A more precise classification of patients with $\mathrm{AF}$ that recognizes and accounts for the major disease mechanisms is needed. Characterizing the major health modifiers causing AF would provide the basis for tailored management, thereby yielding a maximum benefit and limiting adverse effects, in comparison with the current 'one-size-fits-all' therapeutic approach. For example, weight reduction can help to prevent AF recurrence 


\section{Box 2 | Candidates for health modifiers of AF \\ - Ageing and replacement of cardiomyocytes with extracellular matrix \\ - Adaptive changes to increased work load \\ - Delayed left atrial activation \\ - Spontaneous atrial electrical activity \\ - Genetic and genomic predisposition for atrial dysfunction \\ - Infiltration of fat cells in the atria and activation of atrial fat tissue \\ - Elevated atrial oxidative stress \\ - Renal dysfunction \\ - Prothrombotic dysregulation \\ - Yet unknown modifiers to be identified}

in overweight patients with $\mathrm{AF}^{47,48}$, and physical activity reduction might contribute to the prevention of $\mathrm{AF}$ in athletes ${ }^{49}$. Specialized, nurse-led, AF clinics improve guideline adherence ${ }^{50}$, and might also prove valuable in targeting risk factors, such as weight reduction, in identifying warning signs and symptoms, and by raising patient awareness of their disease. Upstream therapy might help to prevent $\mathrm{AF}$ in patients with heart failure. Notably, other less obvious major health modifiers that cause AF might not have yet been detected and, therefore, would not be treated via targeted therapy. Identifying the unknown factors, and implementing these into a health-modifier-based characterization in the future, could markedly improve patient care. This Consensus Statement should be considered a starting point.

\section{Defining patient health modifiers of AF}

A list of the major health modifiers causing AF, and of the corresponding clinical markers, would form a valid basis for stratified or personalized approaches to prevention and treatment (BOX 2). We therefore propose, first, to identify the major health modifiers causing AF in patients in a systematic, concerted effort, and second, to generate a pathophysiological classification of patients with $\mathrm{AF}$ using the identified health modifiers.

We have chosen the term 'health modifiers' to refer to the major mechanisms driving AF in patients as they are meant to inform preventive and therapeutic approaches for AF. We selected the major mechanisms of AF on the basis of a group discussion and a review of the literature. The mechanisms described here are thought to be credible drivers for $\mathrm{AF}$ and to have a relevant effect in terms of pathogenic potential and prevalence in patients with $\mathrm{AF}$, thus qualifying as candidates for the major health modifiers causing AF (BOX 2). Clearly, this list is a starting point that needs scientific evaluation.

\section{Mechanisms of AF}

At the molecular and cellular levels, several drivers of AF have been identified, including ageing and atrial fibrosis, abnormal calcium homeostasis, sarcoplasmic reticulum calcium leak, ion-channel dysfunction (genetic or acquired), and autonomic dysfunction (for example, in athletes with elevated vagal tone). In addition, accumulating data support important contributions to the pathogenesis of AF of high levels of oxidative stress, infiltration of fat into the atria, as well as increases in the paracrine activity of atrial adipocytes and maladaptation owing to chronic kidney disease. All these mechanisms of $\mathrm{AF}$ (BOX 1) have the potential to lead to atrial dysfunction (FIG. 1), although the involvement of each candidate for a major health modifier requires further validation.

\section{Atrial fibrosis and ageing}

AF leads to profound structural alterations, including an increased formation of extracellular matrix, deposition of fibrous material, and a marked change in gene expression patterns ${ }^{51}$. Both AF itself and various clinical conditions associated with AF, such as inflammation, hypertension, cardiac hypertrophy, or mitral valve disease, can cause increased atrial fibrosis resulting in different fibrotic patterns ${ }^{21,23}$. In addition, the ageing heart is constantly losing cardiomyocytes (estimated at $0.5-1.0 \%$ cardiomyocyte-loss per year ${ }^{52-54}$ ), and fibrous tissue often forms in lieu of cardiomyocytes in older individuals. Impaired electrical coupling between myocytes within the epicardial layer, as well as between the epicardial layer and the endocardial bundle network, fosters three-dimensional, temporospatial conduction events (breakthroughs) ${ }^{55}$, thereby maintaining AF.

\section{Altered calcium homeostasis}

In the presence of $\mathrm{AF}$, high atrial rates and early reactivation of cardiomyocytes elevate diastolic $\mathrm{Ca}^{2+}$ and intracellular $\mathrm{Ca}^{2+}$ storage ${ }^{56,57}$. Adaptation to this new situation results in profound changes in the ion channels controlling $\mathrm{Ca}^{2+}$ reuptake and release by the sarcoplasmic reticulum ${ }^{13,21}$. These changes persist for some time after the restoration of a normal sinus rhythm (for example, after cardioversion), rendering the recurrence of AF more likely ${ }^{58}$. Increases in spontaneous electrical activity have been suggested to have a major role in abnormal intracellular $\mathrm{Ca}^{2+}$ handling in the genesis of $\mathrm{AF}^{59}$. Although this theory has been challenged by reports demonstrating improved $\mathrm{Ca}^{2+}$-handling stability, and even $\mathrm{Ca}^{2+}$ silencing, after $\mathrm{AF}^{56,60}$, increases in the sympathetic activity in the atrial tissue might still underlie the increase in the rate of ectopic activity during AF.

\section{lon-channel dysfunction}

$\mathrm{AF}$ and other structural heart diseases result in changes in the expression, or in the post-translational regulation, of ion channels ${ }^{21}$. These changes not only contribute to a shortening of atrial refractoriness, favouring re-entry, but also to prolongation of the atrial action potential and the triggered electrical activity ${ }^{13,21}$. In addition to these adaptive changes in ion-channel regulation, the local milieu within atrial myocytes - influenced for example by shear stress ${ }^{16}$, metabolic factors, atrial work load, or cellular age - also alters the expression and function of ion channels, possibly contributing to changes in refractoriness or in ectopic activity occurrence ${ }^{61}$. 


\section{Genetic causes}

AF has a strong familial component. Some pedigrees of early-onset AF are driven by mutations in ion-channel genes, which are also found in patients with inherited electrical diseases, such as the long QT syndrome, Brugada syndrome, and hypertrophic or arrhythmogenic right ventricular cardiomyopathy ${ }^{62-64}$. Many inherited arrhythmia syndromes, which are characterized by mutations in cardiac ion channels, cause AF in structurally normal hearts ${ }^{64,65}$. Patients with AF who have these mutations have been referred to as patients with 'monogenic AF' (REF. 1). Early-onset AF is associated with common genetic variants (17 independent loci have been identified to date), with a hotspot for such variants on chromosome 4q25 (REFS 18,66). The gene most closely located to the risk variants encodes the two-domain transcription factor, PITX2. Low expression levels of PITX2 mRNA induce complex left atrial gene expression changes, without apparent structural alterations, that predispose to $\mathrm{AF}^{19,67}$. Thus, altered expression of atrial ion channels as a result of genetic alterations in the atria could be a common path by which subtle genetic changes predispose patients to AF, subsequently influencing the response to antiarrhythmic drugs ${ }^{68}$.

\section{Autonomic dysfunction}

Atrial function is tightly regulated by the autonomic nervous system, which in turn can be an important factor that promotes the new onset of AF. Changes in sympathetic or parasympathetic tone alter the atrial action potential, as well as the refractory period, provoking depolarizations and triggered activity ${ }^{69-71}$. One in three patients with paroxysmal AF, and up to $70 \%$ of younger patients, present with well-defined adrenergic or vagal triggers $^{72,73}$. Low-level vagal stimulation prevents AF by decreasing sympathetic and parasympathetic cardiac responsiveness in animal models susceptible to $\mathrm{AF}^{74}$. High-intensity endurance training is an evolving risk factor underlying AF in middle-aged Europeans without overt structural heart disease $\mathrm{e}^{75,76}$, which can be mediated by an elevated parasympathetic tone, structural changes, or by changes in ion-channel expression ${ }^{14,73,77}$. Similarly, AF in patients with obstructive sleep apnoea can be provoked by autonomic imbalance ${ }^{14,78}$. Ganglionated plexi, heterogeneous sympathetic hyperinnervation, and nerve sprouting can contribute to AF in some patients ${ }^{79,80}$.

\section{Oxidative stress}

Experimental studies have suggested that changes in the nitric oxide-redox balance of the atrial myocardium can

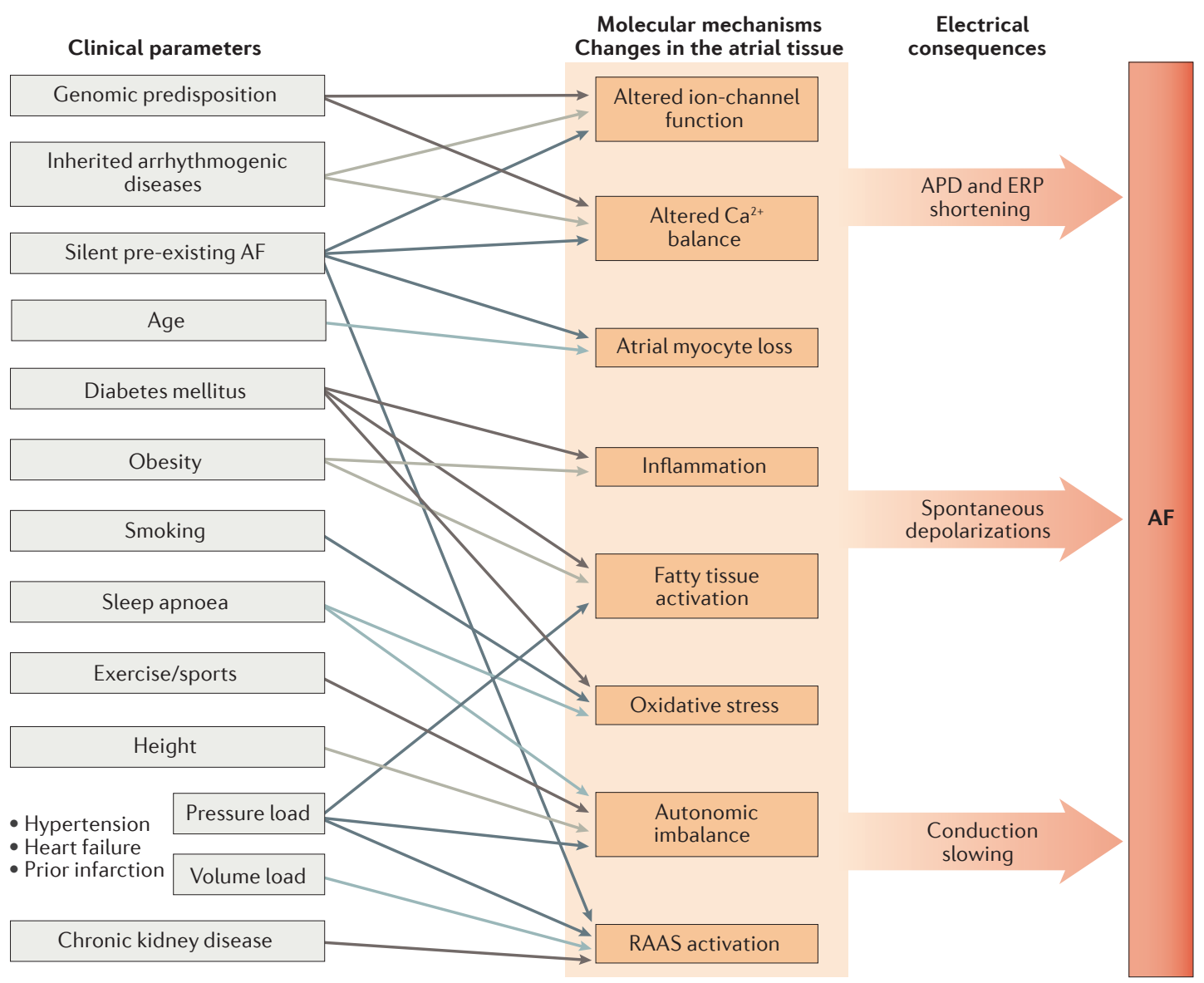

Figure 1 | Hypothetical network of the clinical conditions and mechanisms associated with AF. Proposed interaction between the clinical parameters that have been associated with atrial fibrillation (AF), the known mediators of atrial damage, the dysfunction that might be driven by these conditions (orange), and the major electrical consequences causing $\mathrm{AF}$ (red). APD, action-potential duration; ERP, effective refractory period; RAAS, renin-angiotensin-aldosterone system. 


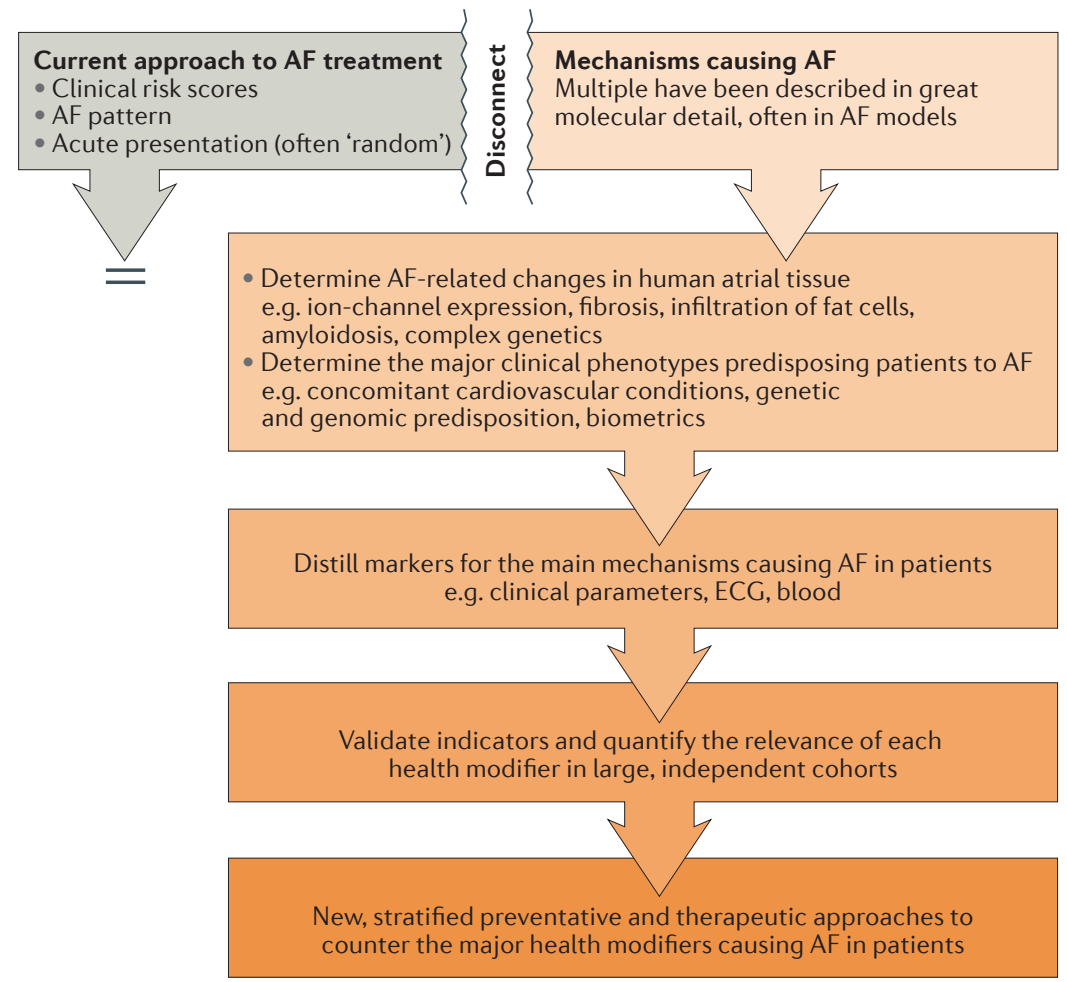

Figure 2 | Disconnect between the mechanisms and the clinical treatment of AF, and the proposed strategy to overcome it. Comparison of the current approach to atrial fibrillation (AF) management, which is disconnected from the established insights into $\mathrm{AF}$ pathophysiology, and the proposed approach to $\mathrm{AF}$ management in which the major changes leading to AF will lead to a classification of patients with $A F$ and provide a basis for personalized prevention and management. ECG, electrocardiogram.

have an important role in the new onset and progression of AF, by mediating the effects of systemic inflammation on the atrial myocardium, and by acting on a number of relevant mechanisms. In humans, the cytokine-stimulated gp91 $1^{\text {phox }}$ NADPH oxidase (Nox2) is the main source of reactive oxygen species in isolated atrial myocytes ${ }^{81}$. Atrial Nox 2 activity was significantly increased shortly after AF induction in goats, and in atrial samples from patients in sinus rhythm who developed AF after cardiac surger ${ }^{20}$. These findings suggest that Nox2 inhibition with statins might prevent the new onset of AF. However, the Statins In Cardiac Surgery trial ${ }^{82}$ has provided good evidence against this assumption, suggesting that either the level of Nox2 inhibition achieved by therapeutic doses of statins in humans is not sufficient to prevent postoperative $\mathrm{AF}$, or that the association between atrial Nox2 activity and AF is not causal.

\section{Fat-cell infiltration and activation}

The profound and swift effects of weight loss on $\mathrm{AF}^{47,48}$ cannot be explained by long-term cardiovascular protection $^{83}$. Obesity not only creates atrial fibrosis ${ }^{84}$, but also increases epicardial fat ${ }^{84,85}$. Furthermore, obesity leads to the infiltration of fat cells into the atrial tissue and to the activation of atrial fat cells, thus modifying atrial electrical function ${ }^{22,86}$, providing a possible mechanism linking obesity and pericardial fat to AF. Therefore, epicardial adipose tissue could be an important health modifier for AF.

\section{Chronic kidney disease}

In large, unselected study populations, the risk of developing $\mathrm{AF}$ is increased in patients with a reduced estimated glomerular filtration rate of $30-59 \mathrm{ml} / \mathrm{min} / 1.73 \mathrm{~m}^{2} \mathrm{com}-$ pared with those with normal renal function (HR 1.32), independently of other risk factors ${ }^{87,88}$. Additionally, microalbuminuria and macroalbuminuria were associated with increased risk of $\mathrm{AF}^{87,88}$. In patients with both chronic kidney disease and AF, the risk of stroke and the risk of bleeding are further increased compared with patients with AF only ${ }^{87,88}$. However, neither vitamin $\mathrm{K}$ antagonist anticoagulant therapies, nor the non-vitamin-K antagonist oral anticoagulants, such as apixaban, dabigatran, edoxaban, or rivaroxaban, have been sufficiently tested in patients with severe chronic kidney disease to suggest safe use at present ${ }^{87,88}$.

\section{From atrial tissue to clinical markers}

Several mechanisms that can cause AF have been identified and verified, but often in animal models of AF. This knowledge requires verification in patients, and translation into clinical markers that can be measured in clinical practice without needing access to atrial tissue (FIG. 2). These mechanisms have plausible links to clinical conditions and to markers that can be used to identify patients at risk of AF (FIG. 1), which provides good reason to explore putative markers for relevant health modifiers for AF. Thereby, a set of clinical markers to define the major health modifiers causing AF in specific patients can be developed, which subsequently will require validation in independent cohorts (FIG. 2). Identification and validation of these markers could underpin new personalized approaches to AF prevention and therapy in the future, and ideally will be accessible in a wide variety of health-care settings. Such a set of markers would also accommodate the concept that several mechanisms can coexist and synergistically promote AF in individual patients.

\section{A new classification of AF: a call to action}

A major, direct benefit of a mechanistic classification of AF would be a personalized therapeutic approach on the basis of the most active processes in each patient, in addition to more accurate disease stratification, as well as better prevention of AF, and detection of silent AF (FIG. 3). By identifying the leading health modifiers causing AF in individual patients, more effective therapies than those currently available could be selected at an earlier stage for primary preventive intervention or for the prevention of AF recurrence (FIG. 2). Such personalized concepts will add to the existing practice of cardiovascular risk reduction. These interventions will comprise targeted treatment of reversible health modifiers, such as antifibrotic treatment in patients prone to atrial fibrosis, weight reduction in patients with atrial fat-cell infiltration, or selection of antiarrhythmic drugs on the basis of the atrial electrical function as determined by age or genetic predisposition ${ }^{36,45}$. Although a complex network of arrhythmogenic processes is likely to culminate in AF, a balance between simplicity and the integration of the major mechanisms of AF is essential. 


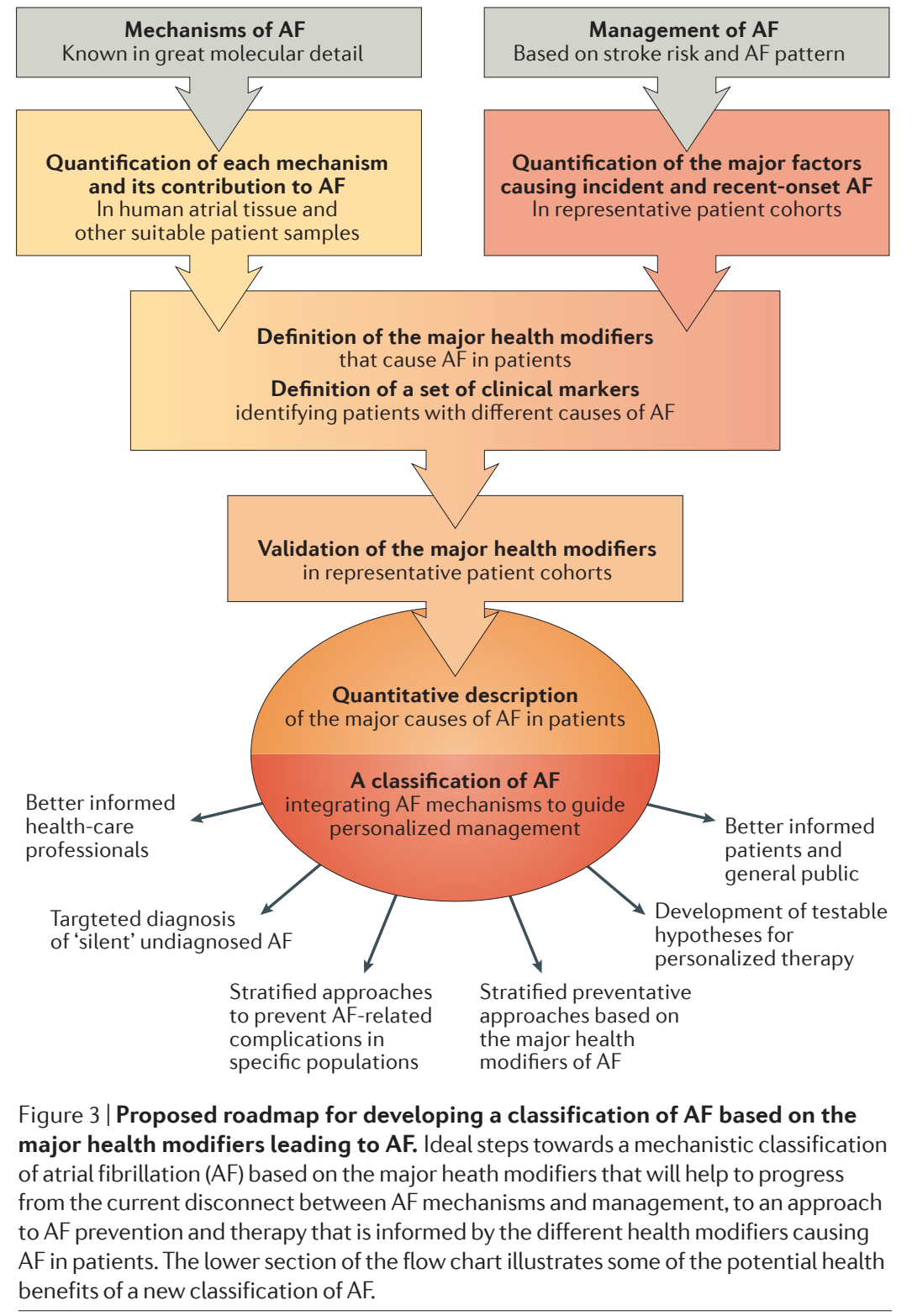

Given the identified potential for personalized prevention and management of $\mathrm{AF}$, scientists, clinician researchers, experts in statistical methods, and those with access to biobanks urgently need to collaborate in characterizing the major health determinants of AF. Most of the pieces in this puzzle are probably available, but we need to put them together by making use of existing biobanks, large databases, as well as interdisciplinary, integrated, and specialist AF expertise. Subsequently, scientific rigour and robust validation of clinical markers for different types of AF are necessary for the new classification of AF to become useful.

The context of health-care delivery is also important. Specific AF services, for example in the form of specialist AF clinics ${ }^{89}$ and integrated AF services ${ }^{50,90}$, will have an important role in the validation and clinical evaluation of the major health modifiers of $\mathrm{AF}^{91}$. Such expert services will also be seminal points for the wider use of the new classification of patients with AF.

\section{Conclusions}

The current clinical approach to patients with AF has yet to consider and incorporate our knowledge on the diverse health modifiers that can cause AF, such as atrial fibrosis and ageing, altered calcium homeostasis, ion-channel dysfunction and genetic susceptibility, autonomic imbalance, oxidative stress, infiltration of fat cells, and chronic kidney disease. A classification of AF reflecting these well-established health modifiers, which could be present alone or in combination, should be able to overcome the current disconnect between our knowledge of the major health modifiers causing $\mathrm{AF}$ and its clinical management, thus informing strategies to personalize the prevention and management of AF in patients. In addition, a deeper knowledge of a patient's disease would enable a betterinformed joint patient-physician decision on which therapeutic strategies to employ. A coordinated effort of researchers from multiple disciplines is warranted to achieve this new, mechanism-based classification of patients with AF.
1. Kirchhof, P. et al. Comprehensive risk reduction in patients with atrial fibrillation: emerging diagnostic and therapeutic options. Thromb. Haemost. 106 1012-1019 (2011).

2. Marijon, E. et al. Causes of death and influencing factors in patients with atrial fibrillation: a competing risk analysis from the Randomized Evaluation of Long-Term Anticoagulant Therapy Study. Circulation 128, 2192-2201 (2013).

3. Lip, G. Y. et al. A prospective survey in European Society of Cardiology member countries of atrial fibrillation management: baseline results of EURObservational Research Programme Atrial Fibrillation (EORP-AF) Pilot General Registry. Europace 16, 308-319 (2014).

4. Kirchhof, P. et al. Management of atrial fibrillation in seven European countries after the publication of the 2010 ESC guidelines on atrial fibrillation: primary results of the PREvention oF thromboemolic events - European Registry in Atrial Fibrillation (PREFER in AF). Europace 16, 6-14 (2014).

5. Kirchhof, P. et al. Improving outcomes in patients with atrial fibrillation: rationale and design of the Early Treatment of Atrial Fibrillation for Stroke Prevention Trial. Am. Heart J. 166, 442-448 (2013).

6. Meinertz, T. et al. Management of atrial fibrillation by primary care physicians in Germany: baseline results of the ATRIUM registry. Clin. Res. Cardiol. 100, 897-905 (2011).

7. Bjorck, S., Palaszewski, B., Friberg, L. \& Bergfeldt, L. Atrial fibrillation, stroke risk, and warfarin therapy revisited: a population-based study. Stroke 44, 3103-3108 (2013)

8. Haim, M. et al. Prospective national study of the prevalence, incidence, management and outcome of a large contemporary cohort of patients with incident non-valvular atrial fibrillation. J. Am. Heart Assoc. 4, e001486 (2015)

9. Schnabel, R. B. et al. 50 year trends in atrial fibrillation prevalence, incidence, risk factors, and mortality in the Framingham Heart Study: a cohort study. Lancet 386, 154-162 (2015).

10. Chugh, S. S., Roth, G. A., Gillum, R. F. \& Mensah, G. A. Global burden of atrial fibrillation in developed and developing nations. Glob. Heart $\mathbf{9}$, 113-119 (2014).

11. Camm, A. J. et al. Guidelines for the management of atrial fibrillation: the Task Force for the Management of Atrial Fibrillation of the European Society of Cardiology (ESC). Europace 12, 1360-1420 (2010)

12. January, C. T. et al. $2014 \mathrm{AHA} / \mathrm{ACC} / \mathrm{HRS}$ guideline for the management of patients with atrial fibrillation: a report of the American College of Cardiology/ American Heart Association Task Force on Practice
Guidelines and the Heart Rhythm Society. J. Am. Coll. Cardiol. 64, e1-e76 (2014)

13. Wakili, R., Voigt, N., Kaab, S., Dobrev, D. \& Nattel, S. Recent advances in the molecular pathophysiology of atrial fibrillation. J. Clin. Invest. 121, 2955-2968 (2011).

14. Guasch, E. et al. Atrial fibrillation promotion by endurance exercise: demonstration and mechanistic exploration in an animal model. J. Am. Coll. Cardiol. 62, 68-77 (2013).

15. Sanna, T. et al. Cryptogenic stroke and underlying atrial fibrillation. N. Engl. J. Med. 370, 2478-2486 (2014).

16. Boycott, H. E. et al. Shear stress triggers insertion of voltage-gated potassium channels from intracellular compartments in atrial myocytes. Proc. Natl Acad. SCi. USA 110, E3955-E3964 (2013).

17. Dawson, K. et al. MicroRNA29: a mechanistic contributor and potential biomarker in atrial fibrillation. Circulation 127, 1466-1475 (2013).

18. Ellinor, P. T. et al. Meta-analysis identifies six new susceptibility loci for atrial fibrillation. Nat. Genet. 44 670-675 (2012).

19. Kirchhof, P. et al. PITX2c is expressed in the adult left atrium, and reducing Pitx2c expression promotes atrial fibrillation inducibility and complex changes in gene expression. Circ. Cardiovasc. Genet. 4 123-133 (2011) 
20. Reilly, S. N. et al. Atrial sources of reactive oxygen species vary with the duration and substrate of atrial fibrillation: implications for the antiarrhythmic effect of statins. Circulation 124, 1107-1117 (2011).

21. Schotten, U., Verheule, S., Kirchhof, P. \& Goette, A Pathophysiological mechanisms of atrial fibrillation: a translational appraisal. Physiol. Rev. 91, 265-325 (2011).

22. Venteclef, N. et al. Human epicardial adipose tissue induces fibrosis of the atrial myocardium through the secretion of adipo-fibrokines. Eur. Heart J. 36 795-805 (2015).

23. Hu, Y. F., Chen, Y. J., Lin, Y. J. \& Chen, S. A. Inflammation and the pathogenesis of atrial fibrillation. Nat. Rev. Cardiol. 12, 230-243 (2015).

24. Jackson, N. et al. Improving clinical trials for cardiovascular diseases: a position paper from the Cardiovascular Round Table of the European Society of Cardiology. Eur. Heart J. http://dx.doi.org/10.1093/ eurhearti/ehv213.

25. Wynn, G. J. et al. The European Heart Rhythm Association symptom classification for atrial fibrillation: validation and improvement through a simple modification. Europace 16, 965-972 (2014).

26. Charitos, E. I., Purerfellner, H., Glotzer, T. V. \& Ziegler, P. D. Clinical classifications of atrial fibrillation poorly reflect its temporal persistence: insights from 1,195 patients continuously monitored with implantable devices. J. Am. Coll. Cardiol. 63, 2840-2848 (2014).

27. Verma, A. et al. Evaluation and reduction of asymptomatic cerebral embolism in ablation of atrial fibrillation, but high prevalence of chronic silent infarction: results of the evaluation of reduction of asymptomatic cerebral embolism trial. Circ. Arrhythm. Electrophysiol. 6, 835-842 (2013).

28. Pedersen, O. D., Bagger, H., Kober, L. \& Torp-Pedersen, C. Trandolapril reduces the incidence of atrial fibrillation after acute myocardial infarction in patients with left ventricular dysfunction. Circulation 100, 376-380 (1999).

29. Vermes, E. et al. Enalapril decreases the incidence of atrial fibrillation in patients with left ventricular dysfunction: insight from the Studies Of Left Ventricular Dysfunction (SOLVD) trials. Circulation 107, 2926-2931 (2003)

30. Disertori, M. et al. Valsartan for prevention of recurrent atrial fibrillation. N. Engl. J. Med. 360 1606-1617 (2009).

31. Goette, A. et al. Angiotensin II-antagonist in paroxysmal atrial fibrillation (ANTIPAF) trial. Circ. Arrhythm. Electrophysiol. 5, 43-51 (2012).

32. Singhal, R. et al. Colchicine suppresses atrial fibrillation in failing heart. Int. J. Cardiol. 176 651-660 (2014)

33. Deftereos, S. et al. Colchicine for prevention of early atrial fibrillation recurrence after pulmonary vein isolation: a randomized controlled study. J. Am. Coll. Cardiol. 60, 1790-1796 (2012).

34. Imazio, M. et al. Colchicine for prevention of postpericardiotomy syndrome and postoperative atrial fibrillation: the COPPS-2 randomized clinical trial. JAMA 312, 1016-1023 (2014)

35. Antoniades, C. et al. Myocardial redox state predicts in-hospital clinical outcome after cardiac surgery effects of short-term pre-operative statin treatment J. Am. Coll. Cardiol. 59, 60-70 (2012).

36. Kirchhof, P. et al. The continuum of personalized cardiovascular medicine: a position paper of the European Society of Cardiology. Eur. Heart J. 35 3250-3257 (2014).

37. McGann, C. J. et al. New magnetic resonance imaging-based method for defining the extent of left atrial wall injury after the ablation of atrial fibrillation. J. Am. Coll. Cardiol. 52, 1263-127 (2008).

38. Malcolme-Lawes, L. C. et al. Automated analysis of atrial late gadolinium enhancement imaging that correlates with endocardial voltage and clinical outcomes: a 2-center study. Heart Rhythm 10 , 1184-1191 (2013)

39. Bisbal, F. et al. CMR-guided approach to localize and ablate gaps in repeat $\mathrm{AF}$ ablation procedure. JACC Cardiovasc. Imaging 7, 653-663 (2014).

40. Marrouche, N. F. et al. Association of atrial tissue fibrosis identified by delayed enhancement MRI and atrial fibrillation catheter ablation: the DECAAF study. JAMA 311, 498-506 (2014).

41. Andreu, D. et al. Contact force threshold for permanent lesion formation in atrial fibrillation ablation: a cardiac magnetic resonance-based study to detect ablation gaps. Heart Rhythm http:// dx.doi.org/10.1016/j.hrthm.2015.08.010.

42. Sramko, M. et al. Clinical value of assessment of left atrial late gadolinium enhancement in patients undergoing ablation of atrial fibrillation. Int. J. Cardiol 179, 351-357 (2015).

43. Patton, K. K. et al. N-terminal pro-B-type natriuretic peptide as a predictor of incident atrial fibrillation in the Multi-Ethnic Study of Atherosclerosis: the effect of age, sex and ethnicity. Heart 99, 1832-1836 (2013).

44. Chao, T. F. et al. CHADS2 score and risk of new-onset atrial fibrillation: a nationwide cohort study in Taiwan. Int. J. Cardiol. 168, 1360-1363 (2013)

45. Kirchhof, P. et al. Personalized management of atrial fibrillation: proceedings from the fourth Atrial Fibrillation competence NETwork/European Heart Rhythm Association consensus conference. Europace 15, 1540-1556 (2013)

46. Kirchhof, P. et al. Short-term versus long-term antiarrhythmic drug treatment after cardioversion of atrial fibrillation (Flec-SL): a prospective, randomised, open-label, blinded endpoint assessment trial. Lancet 380, 238-246 (2012).

47. Abed, H. S. et al. Effect of weight reduction and cardiometabolic risk factor management on symptom burden and severity in patients with atrial fibrillation: a randomized clinical trial. JAMA 310, 2050-2060 (2013).

48. Pathak, R. K. et al. Long-term effect of goal-directed weight management in an atrial fibrillation cohort: a long-term follow-up study (LEGACY). J. Am. Coll. Cardiol. 65, 2159-2169 (2015).

49. Heidbuchel, H. et al. Endurance sports is a risk factor for atrial fibrillation after ablation for atrial flutter. Int. J. Cardiol. 107, 67-72 (2006).

50. Hendriks, J. M. et al. Nurse-led care versus usual care for patients with atrial fibrillation: results of a randomized trial of integrated chronic care versus routine clinical care in ambulatory patients with atrial fibrillation. Eur. Heart J. 33, 2692-2699 (2012).

51. Hsu, J. et al. Whole genome expression differences in human left and right atria ascertained by RNA sequencing. Circ. Cardiovasc. Genet. 5, 327-335 (2012).

52. Spach, M. S., Heidlage, J. F., Dolber, P. C $£$ Barr, R. C. Mechanism of origin of conduction disturbances in aging human atrial bundles: experimental and model study. Heart Rhythm 4 175-185 (2007).

53. Spach, M. S., Heidlage, J. F., Dolber, P. C. $\S$ Barr, R. C. Electrophysiological effects of remodeling cardiac gap junctions and cell size: experimental and model studies of normal cardiac growth. Circ. Res. 86, 302-311 (2000).

54. Spach, M. S., Dolber, P. C.\& Heidlage, J. F. Interaction of inhomogeneities of repolarization with anisotropic propagation in dog atria. A mechanism for both preventing and initiating reentry. Circ. Res. 65, 1612-1631 (1989).

55. Eckstein, J. et al. Time course and mechanisms of endo-epicardial electrical dissociation during atrial fibrillation in the goat. Cardiovasc. Res. 89, 816-824 (2011).

56. Greiser, M. et al. Tachycardia-induced silencing of subcellular $\mathrm{Ca}^{2+}$ signaling in atrial myocytes. J. Clin. Invest. 124, 4759-4772 (2014).

57. Voigt, N. et al. Cellular and molecular mechanisms of atrial arrhythmogenesis in patients with paroxysmal atrial fibrillation. Circulation 129 , 145-156 (2014).

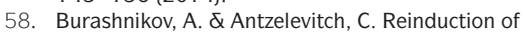 atrial fibrillation immediately after termination of the arrhythmia is mediated by late phase 3 early afterdepolarization-induced triggered activity. Circulation 107, 2355-2360 (2003).

59. Heijman, J., Voigt, N., Nattel, S. \& Dobrev, D. Cellular and molecular electrophysiology of atrial fibrillation initiation, maintenance, and progression. Circ. Res. 114, 1483-1499 (2014).

60. Christ, T. et al. Arrhythmias, elicited by catecholamines and serotonin, vanish in human chronic atrial fibrillation. Proc. Natl Acad. Sci. USA 111, 11193-11198 (2014).

61. Balse, E. et al. Dynamic of ion channel expression at the plasma membrane of cardiomyocytes. Physiol. Rev. 92, 1317-1358 (2012)

62. Camm, C. F. et al. Prevalence of atrial arrhythmias in arrhythmogenic right ventricular dysplasia/ cardiomyopathy. Heart Rhythm 10, 1661-1668 (2013).
63. Eckardt, L. et al. Brugada syndrome and supraventricular tachyarrhythmias: a nove association? J. Cardiovasc. Electrophysiol. 12 680-685 (2001)

64. Zellerhoff, S. et al. Atrial arrhythmias in long-QT syndrome under daily life conditions: a nested case control study. J. Cardiovasc. Electrophysiol. 20, 401-407 (2009)

65. Kirchhof, P., Zellerhoff, S., Mönnig, G. \& Schulze-Bahr, E. Pauses after burst pacing provoke afterdepolarizations and torsades de pointes in a patient with long QT syndrome. Heart Rhythm 1, 720-723 (2004)

66. Gudbjartsson, D. F. et al. Variants conferring risk of atrial fibrillation on chromosome 4q25. Nature 448, 353-357 (2007)

67. Wang, J. et al. Pitx2 prevents susceptibility to atrial arrhythmias by inhibiting left-sided pacemaker specification. Proc. Natl Acad. Sci. USA 107, 9753-9758 (2010).

68. Parvez, B. et al. Symptomatic response to antiarrhythmic drug therapy is modulated by a common single nucleotide polymorphism in atrial fibrillation. J. Am. Coll. Cardiol. 60, 539-545 (2012).

69. Liu, L. \& Nattel, S. Differing sympathetic and vagal effects on atrial fibrillation in dogs: role of refractoriness heterogeneity. Am. J. Physiol. 273, H805-H816 (1997).

70. Sharifov, O. F. et al. Roles of adrenergic and cholinergic stimulation in spontaneous atrial fibrillation in dogs. J. Am. Coll. Cardiol. 43, 483-490 (2004).

71. Patterson, E., Po, S. S., Scherlag, B. J. \& Lazzara, R. Triggered firing in pulmonary veins initiated by in vitro autonomic nerve stimulation. Heart Rhythm 2, 624-631 (2005).

72. Calvo, N. et al. Emerging risk factors and the doseresponse relationship between physical activity and lone atrial fibrillation: a prospective case-control study. Europace http://dx.doi.org/10.1093/europace euv216.

73. de Vos, C. B. et al. Autonomic trigger patterns and anti-arrhythmic treatment of paroxysmal atrial fibrillation: data from the Euro Heart Survey. Eur. Heart J. 29, 632-639 (2008).

74. Shen, M. J. et al. Continuous low-level vagus nerve stimulation reduces stellate ganglion nerve activity and paroxysmal atrial tachyarrhythmias in ambulatory canines. Circulation 123, 2204-2212 (2011).

75. Drca, N., Wolk, A., Jensen-Urstad, M. \& Larsson, S. C. Atrial fibrillation is associated with different levels of physical activity levels at different ages in men. Heart 100, 1037-1042 (2014)

76. Molina, L. et al. Long-term endurance sport practice increases the incidence of lone atrial fibrillation in men: a follow-up study. Europace 10, 618-623 (2008).

77. D'Souza, A et al. Exercise training reduces resting heart rate via downregulation of the funny channel HCN4. Nat. Commun. 5, 3775 (2014).

78. Ramos, P. et al. Atrial fibrosis in a chronic murine model of obstructive sleep apnea: mechanisms and prevention by mesenchymal stem cells. Respir. Res. 15, 54 (2014)

79. Katritsis, D. G. et al. Autonomic denervation added to pulmonary vein isolation for paroxysmal atrial fibrillation: a randomized clinical trial. J. Am Coll. Cardiol. 62, 2318-2325 (2013).

80. Nishida, K. et al. The role of pulmonary veins versus autonomic ganglia in different experimental substrates of canine atrial fibrillation. Cardiovasc. Res. 89, 825-833 (2011).

81. Kim, Y. M. et al. A myocardial Nox2 containing $\mathrm{NAD}(\mathrm{P}) \mathrm{H}$ oxidase contributes to oxidative stress in human atrial fibrillation. Circ. Res. 97, 629-636 (2005).

82. Casadei, B. "Statins In Cardiac Surgery" Hotline. Presented at the European Society of Cardiology Congress 2014

83. Charakida, M. et al. Lifelong patterns of BMI and cardiovascular phenotype in individuals aged 60-64 years in the 1946 British birth cohort study: an epidemiological study. Lancet Diabetes Endocrinol. 2, 648-654 (2014)

84. Mahajan, R. et al. Electrophysiological, electroanatomical, and structural remodeling of the atria as consequences of sustained obesity. J. Am. Coll. Cardiol. 66, 1-11 (2015) 
85. Thanassoulis, G et al. Pericardial fat is associated with prevalent atrial fibrillation: the Framingham Heart Study. Circ. Arrhythm. Electrophysiol. 3 345-350 (2010)

86. Chilukoti, R. K. et al. Atrial fibrillation and rapid acute pacing regulate adipocyte/adipositas-related gene expression in the atria. Int. J. Cardiol. 187, 604-613 (2015).

87. Watanabe, H. et al. Close bidirectional relationship between chronic kidney disease and atrial fibrillation: the Niigata preventive medicine study. Am. Heart $J$. 158, 629-636 (2009).

88. Alonso, A. et al. Chronic kidney disease is associated with the incidence of atrial fibrillation: the Atherosclerosis Risk in Communities (ARIC) study. Circulation 123, 2946-2953 (2011).

89. Berti, D. et al. A proposal for interdisciplinary, nurse-coordinated atrial fibrillation expert programmes as a way to structure daily practice. Eur. Heart J. 34, 2725-2730 (2013).

90. Stewart, S. et al. Standard versus atrial fibrillation specific management strategy (SAFETY) to reduce recurrent admission and prolong survival: pragmatic, multicentre, randomised controlled trial. Lancet 385 775-784 (2015)

91. Kirchhof, P. et al. A roadmap to improve the quality of atrial fibrillation management: proceedings from the fifth Atrial Fibrillation Network/European Heart Rhythm Association consensus conference. Europace http://dx.doi.org/10.1093/europace/euv304.

Acknowledgements

Authors involved in this work have received funding from the European Union's Horizon 2020 research and innovation programme, under grant agreement No. 633193 [CATCH ME]

\section{Author contributions}

L.F. and E.G. contributed equally to this Consensus Statement L.F., E.G., G. Breithardt, D.C., B.C., L.M., M.F.S., U.S., M.S. and P.K. researched data for the article. L.F., E.G. G. Breithardt, A.J.C., B.C., H.C., S.H., F.H.-L., S.K., L.M., H.R., M.F.S., U.S. M.S., and P.K. made substantial contributions to the discussion of content. L.F., E.G., E.B., G. Breithardt, B.C.
L.M. U.S., and P.K. contributed to writing the article. L.F. E.G., C.A., I.B., G. Benninger, T.R.B., G. Breithardt, G.B.-S A.J.C., D.C., B.C., W.W.L.C., H.J.G.M.C., J.D., S.H., S.K., N.M. S.M., L.M., H.R., U.S., T.S., M.S., P.V., R.W., A.W., A.Z., and P.K. reviewed and edited the manuscript before submission.

Competing interests statement

All authors except E.G., C.A., I.B., D.C., W.W.L.C., S.M., M.F.S., T.S., and A.W. declare competing interests. See the article online for full details of the relationships.

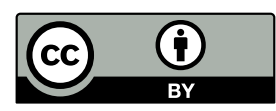

This work is licensed under a Creative Commons Attribution 4.0 International License. The images or other third party material in this article are included in the article's Creative Commons license, unless indicated otherwise in the credit line; if the material is not included under the Creative Commons license, users will need to obtain permission from the license holder to reproduce the material. To view a copy of this license, visit http://creativecommons.org/licenses/by/4.0/. 